\title{
Un enfoque empírico-teológico. En el método, el secreto de Medellín
}

\author{
Carlos Schickendantz \\ CENTRO TEOLÓGICO MANUEL LARRAÍN \\ UNIVERSIDAD ALBERTO HURTADO \\ cschickend@uahurtado.cl
}

Resumen: El método Ver - Juzgar - Actuar revalorizado en Gaudium et spes fue el hilo conductor de los trabajos de la Conferencia de Medellín. El presente artículo desea colaborar a una profundización de esta forma de proceder, que ha sido clave en los procesos eclesiales en nuestro continente. En primer lugar, presta atención a los dos textos oficiales de preparación a Medellín, el Documento Básico Preliminar y el Documento de Trabajo. En un segundo momento, analiza el aporte de Marcos McGrath. Debido a su especial responsabilidad en la etapa de formación de la Constitución pastoral, en sus publicaciones se visibilizan, quizás mejor que en ningún otro protagonista, los resultados del debate metodológico conciliar y su transición hacia el evento de Medellín, con sus nuevas peculiaridades regionales, logros y limitaciones.

Palabras clave: Marcos McGrath, signos de los tiempos, Gaudium et spes

Abstract: The See - Judge - Act method revalued in Gaudium et spes was the guiding thread of the work of the Medellín Conference. This article wishes to contribute to a deepening of this way of proceeding, key to the ecclesial processes in our continent. It starts by focusing on the two official texts of preparation for Medellin, the Basic Preliminary Document and the Working Document. Whereupon it examines the contribution of Marcos McGrath, in whose publications the results of the Vatican II methodological debate and its transition towards the Medellin event - with its new regional peculiarities, achievements and limitations - are better visible perhaps than in those of any other protagonist.

Keywords: Marcos McGrath, Signs of the Times, Gaudium et spes 
Al conmemorarse los treinta y, luego, los cuarenta años de la Conferencia de Medellín José Óscar Beozzo, probablemente el historiador latinoamericano más relevante en los estudios sobre el Vaticano II, afirmó, en las dos oportunidades, que "en el método, (está) el secreto de Medellín"1. En el texto de 2008 escribe: "El método, Ver, Juzgar y Actuar, heredado de la JOC de Joseph Cardijn, se inspiró también en la teología de los «Signos de los Tiempos» de Gaudium et spes y fue el hilo conductor de todos los trabajos de Medellín”. Y porque esa forma de proceder no solo configuró los textos, sino la llamada "mecánica de trabajo" de toda la Conferencia, Beozzo precisa en el artículo de 1998: "Lo que diferenció profundamente el Vaticano II de Medellín fue el método de trabajo adoptado”. Es claro que, con la palabra "secreto", el historiador de San Pablo no pretende aludir a una realidad reservada u oculta a los ojos de los protagonistas de entonces o a los de sus lectores/as actuales, sino más bien, interpreto, a una clave que, en buena medida, explica el evento de Medellín, precisamente como evento, no solo sus textos, sino el significado y simbolismo que progresivamente ha adquirido en la historia posterior. Hay "un antes y un después en la vida de la Iglesia de este continente" ${ }^{2}$.

Es posible encontrar un argumento convergente en otro protagonista destacado de estas décadas, perito en la Conferencia de Medellín, además. Es interesante la figura que usa Gustavo Gutiérrez en su prefacio a la obra de Silvia Scatena, quizás el trabajo histórico de conjunto más relevante sobre aquellos años y sobre el desarrollo de la misma Conferencia. Después de destacar la importancia del "método de aproximación a la realidad" y su "contribución decisiva" para que fuera asumido como forma de trabajo por las más diversas instancias eclesiales posteriores, Gutiérrez afirma que "a esta impostación se ha tratado de volver en Aparecida, como se busca el regreso a las "fuentes»"3. Es significativo que la expresión "fuentes" y la idea de regreso a ellas, un principio clave de renovación del Vaticano II junto al de aggiornamento (PC 2), caracterice

\footnotetext{
J. O. Beozzo, "Medellín: inspiração e raízes", en www.servicioskoinonia.org/relat/202.htm. (original en Revista eclesiastica brasileira 232 (1998) 822-850); id, "Medellin: Quarenta annos", http://www.catedralsaojose.org.br/catedral2011/ reflexao/2534-medellin,-quarenta-anos:-1968-2008.html

G. Gutiérrez, “Actualidad de Medellín”, en Páginas 152 (1998) 6-17, 6.

Cf. "Prefazione", en S. SCATENA, In populo pauperum. La Chiesa latinoamericana dal Concilio a Medellín (1962-1968) (Il Mulino, Bologna 2007) XI-XV, XII (cursiva mía).
} 
aquí al mismo método. Es difícil encontrar una forma más elocuente para describir teológicamente la importancia de esta forma de proceder: "fuente". Este método reclama "la necesidad de partir de la realidad del pueblo en medio del cual la Iglesia estaba llamada a ser un signo visible del Reino"; "consistía en una lectura y un discernimiento de los signos de los tiempos a la luz del mensaje evangélico" ${ }^{4}$. Decisiva es la "aproximación a la realidad", el primer momento, el Ver ${ }^{5}$, pero también el círculo hermenéutico entre los tres momentos que, implícita o explícitamente, se condensan en la noción bíblica y pastoral de signos de los tiempos ${ }^{6}$.

Cuando en 2018 se cumplen cincuenta años de aquella gran Conferencia parece oportuno volver a las "fuentes" (Gutiérrez), su método, y explorar una vez más el "secreto" (Beozzo) de aquel evento que fue caracterizado por varios de sus protagonistas con una imagen ya aplicada por Juan XXIII al Vaticano II: "Dejamos Medellín inspirados como por un nuevo Pentecostés", recuerda Marcos McGrath en $1971^{7}$.

En el marco de la abundante bibliografía existente quiero ofrecer aquí dos aportes concretos, con particular atención al asunto metodológico aludido por Beozzo y Gutiérrez, el "enfoque empírico-teológico" en palabras de McGrath en 1966. En primer lugar, este artículo presta atención a los dos textos oficiales de preparación a Medellín -el Docu-

4 Cf. G. Gutiérrez, "Actualidad de Medellín”, 11: "Si encontrarse dentro del arco temporal del Concilio le da actualidad a Medellín, ella le viene también de haber iniciado en América Latina una forma de ver la realidad y la presencia de Dios en ella: estar atentos a los signos de los tiempos." Casi en los mismos términos, cf. G. Gutiérrez, "El tiempo dirá si los textos de Medellín son letra muerta", en CELAM 2, 19 (1969) 14-15, 14.

5 Escribe G. GutiérRez en marzo de 1969: "El lenguaje empleado en los textos de Medellín puede sorprender. No estamos en verdad acostumbrados a ver al magisterio de la Iglesia empuñar tan firmemente la realidad, seguirla tan de cerca, emplear los mismos términos que usa todo el mundo para hablar de ella. Pero es que el primer momento en el discernimiento de los signos de los tiempos es verlos tal como se presentan a los ojos de los hombres de hoy. Es una condición de veracidad. No hacerlo es viciar el proceso desde el inicio". Cf. "El tiempo dirá", 14.

6 Perspectiva hermenéutica que Gutiérrez adjudica a Juan XXIII, "Actualidad de Medellín", 9: "llama simultáneamente a la sensibilidad frente a las cuestiones del momento y a ahondar en el tesoro de la revelación bíblica. No son tareas que puedan ir por cuerdas separadas. [...] Se trata, pues, de una relación fecunda, de un verdadero círculo hermenéutico...”. Cf. "El tiempo dirá”, 14.

7 Cf. "A Living Theology in Latin America", en The Review of Politics 33, 2 (1971) 163-171, 168. 
mento Básico Preliminar (DBP), de enero de 1968 y, el Documento de Trabajo (DT), de junio del mismo año-, especialmente a la Introducción general del primero de ellos. En un segundo momento, es objetivo de esta contribución detenerse, más de lo que se ha hecho hasta ahora, en el aporte de Marcos McGrath, en particular, en el aspecto metodológico. En el Vaticano II el obispo panameño fue presidente de una de las dos subcomisiones responsables de la preparación de Gaudium et spes en las fases finales del Concilio; la doctrinal-dogmática era dirigida por G. Garrone, la de signos de los tiempos, por McGrath. Fue el encargado de presentar a la asamblea conciliar, en el último período de sesiones, el informe oficial correspondiente a la parte introductoria del texto de la futura Constitución y de explicar el significado que asumía la categoría signos de los tiempos en el documento ${ }^{8}$. Posteriormente, como dirigente del CELAM McGrath participó durante todo el proceso de preparación a Medellín, fue el encargado de la primera ponencia de la Conferencia, sobre "los signos de los tiempos en América Latina hoy", se desempeñó, además, como presidente de la Comisión $n^{\circ} 5$ - Educación de la fe y, en ella, de la Subcomisión B) Pastoral de las elites, que formuló, precisamente, las expresiones más detalladas del documento final sobre los signos de los tiempos 9 . Por tanto, debido a su especial responsabilidad en la etapa de formación de Gaudium et spes, en sus intervenciones y publicaciones, en especial en las de aquellos años, se visibilizan, quizás mejor que en ningún otro protagonista de Medellín, los logros y los límites del debate metodológico conciliar y su transición hacia el evento de Medellín, con sus nuevas peculiaridades regionales, también con sus logros y limitaciones. Podría decirse que McGrath representa una vía de acceso privilegiada para una mejor comprensión del "secreto" de Medellín ${ }^{10}$.

8 Cf. C. Schickendantz, "Una elipse con dos focos. Hacia un nuevo método teológico a partir de Gaudium et spes", en Teología 110 (2013) 85-109, 87-89, 97-101.

9 "Esta evangelización debe estar en relación con los «signos de los tiempos». No puede ser atemporal ni ahistórica. En efecto, los «signos de los tiempos» que en nuestro continente se expresan sobre todo en el orden social, constituyen un «lugar teológico» e interpelaciones de Dios." (Elites 7, I, 13).

10 Una breve descripción de la biografía de M. McGrath ofrece P. Casarella, "En busca del pasado y futuro del laicado. Proyecciones latinas a partir del testimonio de Marcos McGrath y Lucio Gera”, en Atualidade Teológica 52 (2016) 77-105, 83ss, 98-100. Como afirma J. WiCKS, sería de desear que, de forma análoga a las producciones hechas en Bélgica, por ejemplo, se publicaran elencos bibliográficos 


\section{El Documento Básico Preliminar y el Documento de Trabajo}

1.1. Con una carta fechada el 19 de febrero de 1968, Avelar Brandao Vilela, arzobispo de Teresiña y presidente del CELAM, envió a las conferencias episcopales de todo el continente el "texto básico preliminar" que "fue aprobado, en líneas generales, en la reunión del CELAM de noviembre de 1967 y posteriormente revisado y profundizado en un Encuentro de Expertos convocados por el CELAM" en enero de 1968. Considerado "como texto básico preliminar" fue enviado a todas las conferencias episcopales a fin de que lo sometieran "a un atento examen" y formularan "las sugerencias que puedan enriquecerlo o mejorarlo". Como el tiempo urgía, advertía Brandao Vilela entonces, se solicitaba "enviar la indispensable contribución de opiniones" antes de fin de abril o comienzos de mayo de 1968. El Documento básico preliminar (DBP) poseía 28 páginas mecanografiadas: una introducción general y tres partes; la realidad latinoamericana, reflexión teológica y prioridades pastorales ${ }^{11}$.

El texto tuvo una recepción muy diferenciada por parte de los episcopados $^{12}$. Una crítica común fue la manifiesta desproporción entre las tres partes; en menor medida, pero también comunes fueron las observaciones acerca del tono prevalentemente negativo y pesimista de la primera parte. Por lo demás, el mismo Documento era consciente del asunto; afirma al iniciar la primera parte: "La descripción podrá parecer pesimista, pero es un reflejo de la realidad latinoamericana, que es trágica y que pide una respuesta rápida y definitiva”.

El Documento de trabajo (DT), por su parte, elaborado bajo la coordinación de Mons. Eduardo Pironio y los teólogos Gustavo Gutiérrez, Lucio Gera, Renato Poblete y Pierre Bigo en la primera semana de junio de 1968 en base a las sugerencias y críticas recibidas, "reproducía sustancialmente" el esquema y la articulación del texto elaborado en enero ${ }^{13}$. Pensado inicialmente como un texto reservado para el uso exclusivo de

y papers de, entre otros, M. McGrath, existentes en los archivos de la University of Notre Dame (http://archives.nd.edu/findaids/ead/html/MCG.htm). En este tipo de trabajos, reconoce Wicks, "Estados Unidos está ausente". Cf. "Yet More Light on Vatican Council II", en The Catholic Historical Review 102,1 (2016) 97-117, 99.

11 Publicado más recientemente en una separata especial: Medellín 76 (1993) 1-23.

12 Cf. S. Scatena, In populo pauperum, 313-327.

13 Cf. S. SCATENa, In populo pauperum, 400ss. 
los miembros de la Conferencia, fue hecho público por un diario colombiano. De allí que el presidente del CELAM, Brandao Vilela, en una carta fechada el 24 de julio, les informó a los obispos latinoamericanos que dicho texto era "un simple instrumento de trabajo (...) de ninguna manera, una decisión anticipada a la Conferencia, lo cual sería un absurdo" ${ }^{14}$. Es el testimonio, también, de M. McGrath: "de antemano, se decidió no llegar a Medellín con un texto ya elaborado al que solo se le harían enmiendas" ${ }^{15}$. Las opiniones sobre el DT fueron, naturalmente, variadas ${ }^{16}$, la utilización de ese material en el desarrollo mismo de la Conferencia, bastante modesto.

Para el objetivo de esta contribución, interesa destacar aquí "la estructura vertebral" (Parada), "el esquema jocista" ya bien conocido (Scatena). Como constata H. Parada, el DBP "tenía ya las características que habría de conservar a lo largo de todo su proceso de acontecimiento posterior", ante todo, la de su estructura básica: "la estructura vertebral del documento reproducía, básicamente, el método de "Ver - juzgar actuar»" ${ }^{17}$. La adopción del esquema jocista, pensado como articulación del temario, se puede verificar en la reunión que Scatena caracteriza como "el primer verdadero y propio intercambio en el CELAM sobre la preparación de la conferencia”, en un encuentro de mayo de 1967, si bien analizado luego con más detalle en la reunión de Chaclacayo, Lima, en noviembre de $1967^{18}$.

1.2. El DBP contiene una "Introducción preliminar" de cuatro páginas mecanografiadas en la versión que Brandao Vilela envió a todos los episcopados; incluye aspectos interesantes desde la perspectiva del método. Un asunto que llama la atención es la relectura que se hace del Vaticano II exclusivamente desde Gaudium et spes. Dicha Introducción refiere al Concilio siete veces, solo a la Constitución pastoral. No alude a ninguna otra problemática, ni a ningún otro documento del Concilio.

\footnotetext{
14 S. SCATENa, In populo pauperum, 402.

15 "Algunas reflexiones sobre el impacto y la influencia permanente de Medellín y Puebla en la Iglesia de América Latina”, en Medellín 58-59 (1989) 152-179, 164.

16 Cf. H. Parada, Crónica de Medellín (Indo-American Press Service, Bogotá 1975) 69-72.

17 Crónica de Medellín, 51.

18 Cf. In populo pauperum, 295, 297.
} 
Naturalmente no hay en esas páginas la pretensión de realizar una interpretación global del Concilio, aunque en cierto modo eso ocurre, pero es verdad que este hecho -la focalización exclusiva en ese documento- saca a la luz algo de lo específico de la lectura realizada en aquel momento. Si se pone en conexión esta Introducción con una observación del perito en Medellín, J. Mejía, se obtiene una clave de interpretación de las perspectivas de análisis de, al menos, una parte importante de aquel grupo de personas que estaban ideando la conferencia de Medellín. Afirma Mejía luego de describir la estructura de los documentos finales, concluida la Conferencia: "Un resumen, se diría, de los dieciséis documentos del Segundo Concilio Vaticano, en la perspectiva de Gaudium et spes" ${ }^{\prime \prime}$.

Se materializa en la Introducción general, probablemente, una conciencia explícita acerca de la centralidad de la Constitución pastoral en actores relevantes de aquel momento, como G. Gutiérrez ${ }^{20}$ o M. McGrath. En un texto de 1966, este afirma sobre Gaudium et spes:

"La originalidad de este documento no es un elemento aislado del Concilio. Su terreno de cultivo fue el corazón mismo del Concilio. El Arzobispo Mons. Garrone haría notar en una conferencia de prensa que tuvo cuando la Constitución estuvo lista para su votación final, que este es el documento conciliar que logra con más precisión lo que el Papa Juan deseaba del Concilio y de hecho se podría decir que «es el único esquema expresamente deseado por Juan XXIII»"21.

Precisamente, una referencia relevante del DBP en ese contexto es la del discurso inaugural de Juan XXIII, de octubre de 1962. Se acude a él, sin citarlo literalmente, recordando la idea de la peculiaridad pastoral

19 “El pequeño Concilio de Medellín. II”, en Criterio 1556 (1968) 686-689, 689 (cursiva mía).

20 G. GutiérRez, por ejemplo, en un comentario al documento de Medellín, en marzo de 1969, manifiesta que él es el fruto de una situación nueva; uno de los factores está dado, a su juicio, por "las esperanzas despertadas por el Concilio", que queda caracterizado allí, exclusivamente, en relación a la temática de la Constitución pastoral: "En él la Iglesia toda reafirmó su voluntad de estar plenamente presente en el mundo de hoy. Fiel a lo que el Señor de la historia quiere decirle a través de los acontecimientos que vive nuestra época, la Iglesia emprendió una honda revisión de sí misma.” La referencia a Juan XXIII es una marca registrada del momento. Cf. "El tiempo dirá", 14.

21 M. McGrath, "La génesis de Gaudium et spes”, en Mensaje 153 (1966) 495-502, 501. 
típica del Concilio; esto es, no repetir verdades, ni condenar errores, sino iluminar con la verdad revelada "los cambios de hoy" (DBP). La Introducción evidencia, por tanto, una determinada interpretación del Concilio, además de reflejar cómo imaginan aquel grupo de obispos y peritos, reunidos en noviembre de 1967 y enero de 1968, puntos centrales de la temática y la perspectiva de la Conferencia de Medellín citada para agosto de ese año.

1.3. La Introducción general inicia con una cita: "El género humano se halla en un período nuevo de su historia, caracterizado por cambios profundos y acelerados, que progresivamente se extienden al universo entero" (GS 4b). Los tres aspectos aludidos son importantes para la caracterización del tipo de cambio que se diagnostica, tanto en el Concilio, como en Medellín: profundos, acelerados y globales. Sugestiva es la segunda frase del DBP: "Esta declaración vigorosa del Concilio Vaticano II explica la razón de ser del Concilio mismo" (cursiva mía). Con la perspectiva que ofrecen los trabajos históricos y hermenéuticos hoy existentes, resulta particularmente llamativo que, el motivo del Concilio, "la razón de ser", se manifieste exclusivamente con este argumento, con ese texto y ese contexto.

Que el DBP tomaba la idea del cambio como central se deduce de varias expresiones de la Introducción general: "Todo lo que el Concilio ha dicho sobre este mundo en proceso de cambios rápidos, extensos y profundos, se puede afirmar de manera especial para nuestro mundo latinoamericano". Estos cambios realizan "una transformación" de tal magnitud en las "actitudes y las formas de vida, que debemos hablar de un "nuevo período en su historia»". Una situación calificada como "alarmante", por sus indicadores humanos y sociales; una verdadera "encrucijada de la historia”. El paralelismo con el Vaticano II, la continuidad con él y su desarrollo y especificación ulterior representan una conciencia explícita; el "mundo huius temporis" de Gaudium et spes, es ahora el "mundo latinoamericano" de Medellín: "Como la Iglesia en el Concilio encaró valientemente el nuevo mundo de estos tiempos, así debe la Iglesia en América Latina encarar el nuevo mundo latinoamericano" (DBP. Int.).

Con esta comprensión y este diagnóstico aparece como normal que, inmediatamente, en el segundo párrafo, el documento acuda a GS 4a: escrutar los signos de los tiempos e interpretarlos a la luz del Evangelio. 
Es la única vez que, propiamente, el DBP utiliza la expresión signos de los tiempos, aunque con una traducción del latín errónea: de la "época". Los sinónimos de la expresión en el DBP parecen ser estos términos: la "realidad", en la primera parte, la "situación" al inicio de la segunda y el "diagnóstico" cuando comienza la tercera. Dichos sinónimos dicen algo de la comprensión y recepción de la expresión conciliar signa temporum. El Concilio puso a la Iglesia "en medio del mundo". De allí la tarea que formula el DBP: "en cada lugar y cada momento, este deber permanente de estudiar nuestros tiempos".

1.4. Después de Gaudium et spes, el texto o autor más citado en el DBP es Pablo VI, particularmente en su importante discurso al CELAM de noviembre de 1965, en las semanas finales del Concilio. Un aspecto parece central en la lectura que se hace en este lugar, en la Introducción general del DBP: lo que el texto llama en ese contexto como "examen de la realidad" al servicio de la tarea ya citada de "estudiar nuestros tiempos". "Él (Pablo VI) nos aconseja, en primera instancia, que nos demos cuenta cabal de la realidad en que vivimos y que afecta a la Iglesia. Y para esto nos dice cómo debemos valernos de expertos, sobre todo de sociólogos para el estudio de esa misma realidad"22. El discurso de Pablo VI es relevante por varios aspectos, a los cuales el DBP alude muy sintéticamente. Propone, por ejemplo, la realización de un plan pastoral de conjunto continental. Pero un párrafo del Papa llamaba particularmente la atención, entonces, como se puede verificar también por su cita en el documento de la importante X Asamblea Extraordinaria del CELAM de octubre de $1966^{23}$. Afirmaba Pablo VI en 1965:

“...el Pastor tendrá siempre ojos abiertos al mundo, porque la observancia y la vigilancia evangélica deben continuar, porque el mundo cambia y se necesita ser capaz de satisfacer las demandas crecientes e interpretar las nuevas instancias. Ellos sabrán servirse de la ayuda de

22 También hay que notar la preocupación contraria de algunos episcopados que veían una acentuación excesiva en la perspectiva sociológica en perjuicio de una mirada más creyente y pastoral. Así, por ejemplo, en las respuestas a DBP de los obispos venezolanos, colombianos, argentinos, etc. Cf. S. SCATEnA, In populo pauperum, 313-327.

23 Cf. "Presencia activa de la Iglesia en el desarrollo y en la integración de América Latina”, en Criterio 1520 (1967) 190-195, 190. J. Mejía afirmó que esa alocución al CELAM es un "verdadero programa de renovación pastoral del continente". Cf. "El pequeño Concilio de Medellín”, en Criterio 1555 (1968) 651-653, 652. 
especialistas, teólogos y sociólogos... [...] Para el conocimiento exacto de las situaciones y la urgencia del trabajo, el Pastor hará un uso amplio e inteligente de los datos sociológicos recogidos y elaborados sobre las condiciones religiosas del país" ${ }^{24}$.

El hecho de que la Conferencia se iniciara el 27 de agosto con "una relación fuera de programa" 25 , la del sacerdote brasileño, Affonso Gregory, quien presentó una suerte de "radiografía social" del continente describiendo los principales aspectos de la situación demográfica, económica, social y religiosa tenía en aquella sugerencia de Pablo VI un argumento a su favor. Dicha presentación sirvió de introducción a las ponencias sucesivas ya previstas. El "fuerte impacto" que causó en varios de los participantes está atestiguado por diversas fuentes ${ }^{26}$.

1.5. La "segunda parte" del DBP, titulada simplemente "reflexión teológica”, tiene una extensión y detalle de análisis muy diversa a la primera. Habiendo sido planteada en la primera parte temas de gran envergadura, en los distintos ámbitos, sociales, políticos, económicos, religiosos, o temáticas de tal gravedad como la violencia, el segundo momento, esto es, el "considerar a grandes rasgos esta situación a la luz de la enseñanza de nuestra fe cristiana" es extremadamente genérico. Se esboza una antropología teológica con la ayuda de algunas frases importantes de Gaudium et spes y Lumen gentium. Se habla de la dignidad humana, del proceso de personalización e inserción en una comunidad que tiene su origen en Dios (LG I). Se incluye también una breve reflexión cristológica, su plan de salvación, la realidad del pecado. Se subraya la responsabilidad de los cristianos y de todas las personas en el proceso de cambio. Aquí aparecen varias citas de Populorum progressio.

El segundo momento de esta segunda parte, "Misión específica de la Iglesia", contiene una breve enseñanza sobre algunas de las principales ideas conciliares, con sus citas respectivas: sacramento de salvación, pueblo reunido por Dios y enviado, encargado de anunciar el Reino y mostrar la presencia de Dios en el mundo, de allí una necesaria reforma

24 Cf. http://www.vatican.va/holy_father/paul_vi/speeches/1965/index_sp.htm

25 Cf. S. Scatena, In populo pauperum, 450.

26 Cf. J. O. Beozzo, "Medellin: Vinte annos depois (1968-1988). Depoimentos a partir do Brasil", en Revista Eclesiástica Brasileira 48, 192 (1988) 771-805, 788; S. SCATEna, In populo pauperum, 448. Cf. en el texto de BeOzzo el testimonio del mismo Gregory. Cf. "Medellin: Vinte annos depois”, 785-786. 
de sí misma. En el marco del aporte que ella debe hacer a la sociedad impregnándola con el espíritu evangélico, se dedica un párrafo a "su compromiso de pobreza", atendiendo ante todo a su misma vida interna y, luego, a su compromiso con la sociedad. La carencia de textos y temas bíblicos en todo el DBP es un hecho muy llamativo; diferente es la situación en el DT que, particularmente en la sección antropológica de la segunda parte (n. 11), incluye poco más de una decena de citas neotestamentarias, en su gran mayoría paulinas. Pero, como ha sido observado con acierto, esta perspectiva bíblica permanecerá como un déficit a lo largo de toda la Conferencia, incluido el documento final ${ }^{27}$.

Un aspecto metodológico puede destacarse en el DBP. La referencia que se hace a América Latina en la parte doctrinal, de modo simétrico en los dos puntos -el antropológico y el eclesiológico- y en relación a ideas centrales de ambas partes, probablemente revela la conciencia hermenéutica y el cuidado en los autores del texto por evitar la mera sucesión del momento del Juzgar al momento del Ver, sin que una conexión intrínseca evite que el momento inductivo sea superpuesto, de forma paralela y carente de relación, a una formulación doctrinal a prio$r i$, permanente, no afectada ni interrogada por la lectura contextual que se ha esbozado.

En el DT la estrategia es parcialmente distinta. La conexión entre las partes - "La realidad latinoamericana" y "Reflexión teológica"- se realiza al inicio mismo de la segunda al ofrecer una suerte de resumen de los "datos ya anteriormente señalados" (n. 10) en la primera. Conserva la conexión explícita con la realidad latinoamericana en la reflexión eclesiológica (n. 12.5), prácticamente en los mismos términos que en el $\mathrm{DBP}^{28}$.

27 Cf. J. O. Beozzo, "Medellín: inspiração e raízes", 20-21. Parece que esta debilidad, la carencia bíblica, es común a una gran parte de los textos y discursos en torno a la Conferencia, incluidos, por ejemplo, los textos inaugurales de Pablo VI y de los cardenales Juan Landazuri, A. Samoré y A. Brandao Vilela. La ponencia de Pironio es una excepción.

28 La mayor novedad del DT en relación al DBP reside, quizás, en la descripción del "método de trabajo" (n. 14) propuesto para la Conferencia, que sustancialmente indica las ponencias, que darían comienzo a la asamblea, y la caracterización del "Trabajo de las comisiones pastorales" (n. 15-17), que articularían el segundo momento en el desarrollo de Medellín, con tres secciones o "áreas" como las denominará el documento final (Promoción Humana, Evangelización y crecimiento de la fe, Iglesia visible y sus estructuras) y 9 comisiones con sus subdivisiones que 
1.6. Una modificación importante que se verifica al comparar el DBP de enero y el DT de junio de 1968 es que, en este último, ya no aparece la Introducción general, que hemos comentado. El DT utiliza la expresión "signo de los tiempos", ahora sin referencia a Gaudium et spes, en la introducción a la primera parte ${ }^{29}$. En la segunda parte del DT hay también una referencia implícita a la Constitución pastoral: "Sabe (la Iglesia) también que su propia fe no puede ignorar las manifestaciones de la situación latinoamericana, sino que debe interpretarlas como signos de los tiempos, a la luz del Evangelio" (10.5).

En ninguno de los dos documentos se ofrece una explicación detallada referida a los tres momentos, la "estructura vertebral" (Parada), "el esquema jocista" (Scatena). Aunque es verdad que el tratamiento que se hace en la Introducción general del DBP justifica bien esta forma de proceder, abreviada en el DT. Por otra parte, es al inicio de la tercera parte en el DT que se describe brevemente el triple paso que articula el conjunto: "En la primera parte de este documento se han presentado los grandes rasgos que caracterizan la transformación actual de A. L. y la presencia de la Iglesia, con sus luces y sus sombras. En la segunda parte, se ha reflexionado sobre esa situación del Continente, a la luz de la Palabra revelada, y teniendo en cuenta las orientaciones conciliares y pontificias. Antes de presentar ahora las proyecciones pastorales, que se derivan de las anteriores consideraciones..." (III, 13.1 y 13.2).

Aunque debe destacarse que la conciencia hermenéutica, lo que C. Theobald caracteriza como la "pastoralidad" del Concilio, esto es, la interpretación y reformulación de la tradición a la luz de las exigencias

terminarán por conformar el número de 16 . Según esa caracterización la primera ponencia de McGrath referiría a la primera parte, la encargada a Pironio a la segunda parte, reflexión teológica, y todas las demás a las proyecciones pastorales.

29 "La presencia de nuevos problemas y el planteamiento nuevo de problemas antiguos constituyen un verdadero desafío; pero en el plano de la Providencia se alzan como signo de los tiempos que reclaman imaginación, audacia y trabajo en colaboración para una solución." (DT I, 1.4). Es interesante advertir que el documento final de Medellín, que cita 43 veces a GS -mucho más que cualquier otro documento (le sigue LG con 31) - y que utiliza la expresión signos de los tiempos en 7 lugares -Mensaje a los pueblos; Juventud 5, 13; Elites 7, 13; Catequesis 8, 12; Laicos 10, 13; Sacerdotes 11, 28; Formación del clero 13, 26-, no alude nunca a GS 4 ni a GS 44. Cf. J. O. Beozzo, "Medellín: inspiração e raízes”, 14. 
epocales y contextuales ${ }^{30}$, aflora en múltiples partes de ambos documentos. Un ejemplo entre varios posibles. Al describir la tarea de las futuras comisiones pastorales referidas a la "Iglesia visible y sus estructuras", el DT afirma: "A lo largo de la historia, la Iglesia se ha renovado en su espíritu, revitalizando también sus estructuras, para adecuarlas a las exigencias de cada época. Es lo que ha acontecido, una vez más, en el Concilio Vaticano II" (n. 17.1.). Una reflexión metodológica más específica no se verifica allí.

\section{Un enfolue a la Vez empírico y teológico - Marcos McGrath}

2.1. El momento de "ambientación" inicial ideado por la "mecánica de trabajo" de la Conferencia estuvo conformado, primordialmente, por siete ponencias. La inaugural de M. McGrath, el 27 de agosto de 1968, se comprende mejor a la luz de varios textos del autor escritos en aquella época. Como se ha afirmado en la introducción a esta contribución, sus reflexiones son particularmente importantes a partir de sus responsabilidades y experiencias acumuladas en las difíciles fases finales de la confección de Gaudium et spes.

La historia redaccional de la expresión signa temporum en la Constitución estuvo estrechamente ligada a las discusiones sobre la expositio introductiva y, en general, sobre el método inductivo. El hecho de que en la versión final del documento la expresión solo aparezca una única vez, en el número 4, tiene que ver con los debates que encendió. Ella estuvo ausente en el esquema que ingresó para ser debatido en la última sesión del Concilio, a partir de septiembre de $1965^{31}$. Solo con cuidadas observaciones por parte del relator, M. McGrath, la expresión reingresaría al documento en el textus recognitus, el revisado luego de los debates de septiembre y octubre, distribuido a los padres conciliares en la congregación general 160a, el 13 de noviembre de $1965^{32}$. M. McGrath en la Relatio circa Expositionem introductivam, advierte que "entre los cambios menores", está el del inicio del número 4 que "declara más claramente" la necesidad que la Iglesia tiene de "escrutar los signos de los tiempos e interpretarlos a la luz del Evangelio, ahora como en cualquier

30 Cf. La réception du concile Vatican II. 1. Accéder à la source (Cerf, Paris 2009) 885.

31 Cf. Acta Synodalia Sacrosancti Concilii Vaticani II, IV/1, 435-517.

32 Cf. $A S$ IV/6, 421-560, 425. 
tiempo". Y añade: “Las palabras «signos de los tiempos» utilizadas aquí por única vez, no lo son en sentido técnico, sino general, como se encuentra en varios documentos de los sumos pontífices Juan XXIII y Pablo VI"33. Acogía la preocupación manifestada, incluso por observadores ecuménicos, acerca del sentido preciso, escatológico, que la expresión tiene en la Biblia y, por tanto, diverso al del documento. Es claro que, como consta en el proceso redaccional, la expresión misma tiene vinculación estrecha con Pacem in terris. En el marco de los más de 20.000 modos que se propusieron en esos días y que las diversas comisiones debieron evaluar y resolver, la expresión signa temporum sobrevivió sin dificultades, en un único lugar (GS 4), hasta la votación final del mes de diciembre de 1965.

Visto en perspectiva, en el marco crítico de la tercera sesión y del futuro incierto de la expresión, un momento importante para la evolución de la temática y de la misma fórmula fue la creación, en septiembre de 1964, de dos subcomisiones: una dedicada "para los signos de los tiempos", presidida por M. McGrath, y la otra "de carácter teológico", presidida por G. Garrone. La tarea de la primera "era la de profundizar el análisis del mundo moderno para que el esquema partiera efectivamente de los datos de la realidad histórica. En efecto, la elección del método inductivo era, en este momento, todavía muy incierto y se temía mucho que a la desconfianza de los teólogos se sumase la incomprensión de los padres conciliares" ${ }^{34}$. De allí que una de las principales tareas de la subcomisión consistiera en profundizar el significado del concepto que era comprendido sustancialmente desde una perspectiva fenomenológica: con él se aludía a "fenómenos que por su difusión y por su gran frecuencia caracterizan una época y a través de los cuales encuentran expresión las necesidades y las aspiraciones de la humanidad actual" 35 . Como recuerda el mismo McGrath en 1967, el trabajo de la subcomisión "indujo a componer la introducción expositoria a la Constitución con todo lo que

33 AS IV/6 563-565, 564 (cursiva mía).

34 G. Turbanti, Un Concilio per il mondo moderno. La redazione della costituzione pastorale Gaudium et spes del Vaticano II (Il Mulino Bologna 2000) 373 (cursiva mía). Cf. C. SchicKendantZ, “¿Una transformación metodológica inadvertida? La novedad introducida por Gaudium et spes en los escritos de Joseph Ratzinger”, en Teología y Vida 57 (2016) 9-37.

35 Expresión de François Houtart (perito conciliar belga), citado en G. Turbanti, Un Concilio per il mondo moderno, 374. 
ello significaba para el tono y el método empleados en el curso del documento"; se trata del "acercamiento empírico" que señalaba "la novedad del esquema"; dicha "introducción descriptiva es resueltamente objetiva y hasta fenomenológica" ${ }^{\prime \prime}$.

Las diferencias en el planteo metodológico de Gaudium et spes la formuló M. McGrath en aquellos años con gran claridad, probablemente con una precisión mayor que la de muchos de sus colegas europeos de entonces. En otra contribución he referido ya al breve, pero importante texto suyo de $1966^{37}$. Allí alude McGrath a la disputa acerca del método que, según "los teólogos llamados progresistas", refiere a los que habían redactado Lumen gentium, "debía proceder de acuerdo con el método teológico aceptado, lo que significaba partir de los principios de la revelación, que derivan de las normas de la fe y de la moral, aun para el orden temporal". Esto es, un procedimiento que subordina unilateralmente lo práctico a lo teórico, lo pastoral a lo doctrinal. Por el contrario, afirma, había que "proyectar un nuevo método para este nuevo tipo de documento. Es este nuevo método, desarrollado y perfeccionado muy lentamente, imperfecto aún en el último borrador de la Constitución, el que el lector debe captar si quiere interpretar bien dicha Constitución"38. En una alocución de 1967, McGrath se pregunta, “¿Cuál es este método?”, al que bien "podría llamarse método pastoral doctrinal de esta importante

36 M. McGrath, "Notas históricas sobre la Constitución Pastoral Gaudium et spes", en G. Baraúna (ed.), La Iglesia en el mundo de hoy (Studium ediciones, Madrid 1967) 165-181, 172, 179. McGrath destaca que, incluso en los capítulos de la segunda parte de la constitución, el "progreso real" no está tanto "en su contenido", "ni en la síntesis, o la reunión de todos estos puntos; ni siquiera en el hecho de que el Concilio se interesara con tanto apremio por estos problemas. Está más bien en el modo de abordar los problemas. La descripción empírica de la situación concreta, que comienza en la introducción descriptiva, se renueva al principio de cada nuevo capítulo". Por imperfecta que sea su realización, McGrath "augura actitudes radicalmente nuevas en nuestra teología moral." Cf. "Notas históricas”, 180-181 (cursiva mía).

37 Cf. C. Schickendantz, "Una elipse con dos focos", 87-89.

38 M. McGrath, "La génesis de Gaudium et spes", en Mensaje 153 (1966) 495-502, 496 (cursivas mías). McGrath vincula esta nueva forma de proceder, lo contrario a una "suerte de un moralismo a priori", con el discurso inaugural de Juan XXIII, en su "referencia a "la manera de investigar y de proponer la doctrina» que «requieren nuestros tiempos» es un punto importante y decidor". Cf. "La génesis de Gaudium et spes", 497. 
Constitución"39. Su respuesta es allí directa: "Muy simplemente, el método de investigación, tan bien conocido de los miembros, los moderadores y estudiantes de los movimientos especializados de acción católica de la Iglesia durante muchos años". Añade: "Solo acabado el Concilio, cuando el documento fue finalmente votado y aprobado, podemos decir que este método fue más o menos aplicado con un cierto grado de clari$\mathrm{dad}$, aun cuando deje todavía que desear" ${ }^{40}$. Es notable, afirma, que esta forma de proceder, "esté en marcha en América Latina desde hace más de treinta años y crezca cada vez más” ${ }^{1}$.

2.2. En ese marco se comprenden mejor las reflexiones de la ponencia de McGrath en Medellín, "Los signos de los tiempos en América Latina hoy". Para caracterizar el concepto de signo de los tiempos distingue entre el "lenguaje bíblico" y la "otra forma, más actual", usada por Pacem in terris. Y aclara explícitamente: la Constitución "adoptó el término en este nuevo sentido" ${ }^{42}$. Refiere a las discusiones conciliares y, particularmente, al entonces ya famoso texto de M.-D. Chenu de 1965, añadiendo: "Este artículo muy oportuno sirvió para aclarar el sentido que se daría a este término en Gaudium et spes." El papel muy importante desempeñado por Chenu en aquellas semanas decisivas, aunque él no era perito conciliar y no trabajó directamente en la Constitución, está ya puesto de relieve, particularmente en los trabajos de G. Turbanti y H.-J Sander ${ }^{43}$.

39 "Un nuevo método de pastoral de la Iglesia", en Criterio 1543 (1968) 134-137, 134.

40 "Un nuevo método de pastoral", 134-135 (cursiva mía).

41 "Un nuevo método de pastoral”, 136.

42 En CELAM (ed.), La Iglesia en la actual transformación de América Latina a la luz del Concilio. I. Ponencias (Secretariado General del CELAM, Bogotá 1969) 75-100, 75. Cf. M. McGrath, "Notas históricas", 172: a la subcomisión que presidía en el Concilio, "le competía estudiar la noción de "signos de los tiempos», término usado en el documento a imitación de Juan XXIII" (cursiva mía). De manera análoga se expresa en 1996 y añade: "El concepto sugiere la investigación, el acercamiento al análisis social, la técnica del «ver-juzgar-actuar» de los jocistas, que se ha hecho común en la Iglesia después del Concilio, ampliamente debido a su uso, si bien modificado en esta Constitución." Cf. "El Concilio Vaticano y el futuro", en $\mathrm{Me}$ dellin 86 (1996) 11-35, 25.

43 En los textos de McGrath no parece haber un tratamiento más explícito del contenido del artículo de Chenu; hubiera colaborado para un análisis más preciso del concepto de signos de los tiempos. 
Puede advertirse que, en la ponencia al inicio de Medellín, el término signo de los tiempos en el "lenguaje bíblico", lo "escatológico" para McGrath es solo el evento futuro: "un indicio del fin del mundo y de la segunda venida del Señor", no una irrupción del eschaton en la historia. Por otra parte, los signos de los tiempos según la "otra forma, más actual" de Gaudium et spes es caracterizada, a su juicio, de esta manera: "son en sí aquellos grandes hechos, acontecimientos y actitudes o relaciones que caracteriza a una época”. Y, con una consideración que parece ir un poco más allá, al menos explícitamente, de sus propias expresiones en el Concilio, añade: "Proyectan su significado sobre dos niveles". El primero, que revelan "las corrientes subterráneas", "causas y efectos de los eventos", "las esperanzas y preocupaciones" de los seres humanos. Por ello, quien comprende los signos "puede intuir y palpar... la corriente dinámica de su tiempo", "la historia que vive", así también "podrá mejor influir en ella". El segundo nivel, refiere a la interpretación de la fe: el "creyente trata de intuir más a fondo". "Creyendo en la historia de la salvación, que se plasma en la historia del mundo, él se esfuerza para ver a través de esos signos y las corrientes temporales que revelan, al espíritu de Dios obrando en «el mundo en que vivimos, sus esperanzas, sus aspiraciones y el sesgo dramático que con frecuencia le caracteriza». Esto lo ha de hacer la Iglesia escrutando «a fondo los signos de los tiempos e interpretándolos a la luz del Evangelio»", concluye McGrath citando GS $4^{44}$.

Por tanto, si es posible observar que en los debates conciliares sobre la noción de signos de los tiempos se destacaba más su significado "fenomenológico" o "sociológico", por la pretensión de evitar las observaciones provenientes de la consideración de su significado bíblico, que en la dinámica de la disputa de entonces conducía a excluir el término de la futura Constitución, en la ponencia de Medellín aparece más explícitamente el "doble nivel", empírico y teológico que, no obstante, no recoge la perspectiva que plantea su uso neotestamentario, esto es, cristológico, mesiánico y kairológico (Mt 16,3). Si se revisa su texto de 1966 se corrobora ese doble nivel, sin que se utilice esta expresión, pero carente de toda referencia al significado de la Escritura: afirma la "necesidad de entender los problemas temporales en sus dimensiones propias (...) para poder captar con exactitud la obra de Dios en la creación; y esto no solo

44 Cf. "Los signos de los tiempos", 75-76 (cursiva mía). 
en el comienzo de las cosas, sino también en el "aqui y ahora», y en la figura del mundo que cambia rápidamente al dirigirse hacia el futuro” ${ }^{35}$.

Con la claridad que se advierte en las ideas contenidas en la introducción del DBP, eliminadas luego en el DT, McGrath describe en la ponencia el proceso de la Conferencia de Medellín y su relación con el Concilio:

"Comencemos, como nos lo indica el Concilio, escrutando los signos de los tiempos en América Latina; luego, los consideraremos a la luz del Evangelio; y finalmente trazaremos de esta confrontación algunas proyecciones pastorales para el servicio salvífico de nuestros pueblos. Quizás es poco lo que lograremos esclarecer en estos quince días: pero el hecho de encontrarnos tan seriamente en esta actitud es ya una enorme lección aprendida del Concilio" 46 .

Un aspecto, entre otros, es importante para precisar la comprensión que McGrath exhibe de la relación intrínseca, una suerte de círculo hermenéutico, siquiera de forma inicial, entre los momentos recién descritos. Aclara que el hecho de "distinguir y escrutar los signos... aporta algo nuevo", aunque su "significado no sea de por sí novedoso". ¿Dónde reside, entonces, la relativa "novedad" para McGrath aquí? La describe -implícitamente- en relación a cada uno de los tres momentos. En primer término, "provoca una toma de conciencia de valores" que, aunque ya existían antes, ahora "se perciben con mayor claridad". En este sentido, el "valor no es nuevo; pero la toma de conciencia de él sí lo es". Sin interrupción en el discurso realiza, sin embargo, un paso ulterior hacia el segundo momento: "es así, que los signos de los tiempos nos llevan a decir cosas nuevas del Evangelio", pero el límite de esa novedad se formula con claridad; "no en cuanto a él mismo, pero sí respecto de sus aplicaciones" ${ }^{\prime 7}$. McGrath no avanza más en esta cuestión: la pregunta por la naturaleza exacta de esta "novedad". En relación al tercer momento, McGrath afirma: así también "diremos cosas nuevas para la pastoral aquí en estos días, al responder a las situaciones que los signos de hoy en América Latina nos revelan.” Una reflexión más específica sobre el método, que, por ejemplo, destaque la dimensión cognitiva del actuar, la densidad epistemológica de la praxis, constitutiva y determinante, o,

\footnotetext{
45 M. McGrath, "La génesis de Gaudium et spes", 502 (cursiva mía).

46 "Los signos de los tiempos", 76-77.

47 "Los signos de los tiempos", 77 (cursivas mías).
} 
en general, precise más la circularidad en el ver, juzgar y actuar no se desarrolla aquí. Esta debilidad metodológica, por lo demás, no es exclusiva suya.

Una hipótesis puede formularse aquí: si un aspecto a verificar en todo el proceso de Medellín es la comprensión de la noción de signos de los tiempos, con su implicancia metodológica, pareciera que las especificaciones hechas por McGrath representan la única explicación del concepto. Todos los demás actores, incluido el documento final, hacen uso de la categoría sin problematizarla o hacerla objeto de tratamiento específico. Por otra parte, es verdad que, también McGrath, utiliza el concepto en un sentido más amplio y difuso. En la misma ponencia habla de la Iglesia de América Latina como "un potente signo de estos tiempos" y, en un texto de 1976, califica con esta expresión a la asamblea conciliar: "El Concilio Vaticano II es en sí mismo un signo de los tiempos"48.

2.3. La segunda parte de la ponencia inaugural de McGrath desarrolla "los grandes signos" que él propone a la luz de los múltiples estudios sobre la realidad latinoamericana, del análisis del Concilio, de las orientaciones de Pablo VI y del CELAM. El autor destaca tres, el primero de ellos fundado en su propia experiencia conciliar: "Durante la redacción de Gaudium et spes, se interrogó sistemáticamente a Obispos y peritos de todos los sectores del mundo. A la pregunta, "¿Qué es lo que más caracteriza a su país o región?», invariablemente la respuesta, bajo una u otra forma, fue la misma: el cambio" ${ }^{49}$. Es la lectura que puede apreciarse ya en el DBP, en cuyo inicio, precisamente, está la cita de GS 4 que refiere a "que el género humano se halla hoy en un período nuevo de su historia, caracterizado por cambios profundos y acelerados, que progresivamente se extienden al mundo entero". El DBP afirmaba allí: "Esta declaración vigorosa del Concilio Vaticano II explica la razón de ser del Concilio mismo". Esto se traduce en el título mismo de la Conferencia de Medellín: La Iglesia en la actual transformación de América Latina a la luz del Concilio.

En ese contexto conciliar se comprende la elección de McGrath: "este es, pues, un gran signo de nuestros tiempos, quizá el principal: $e l$ cambio". La tarea que prevé es la comprensión de ese signo general en

48 "Los signos de los tiempos", 99; ID., "Vaticano II: la gran opción”, en Mensaje 246 (1976) 21-34, 25.

49 "Los signos de los tiempos", 79. 
su específica realización latinoamericana: "tomarlo en cuenta, estudiarlo, calcular sus efectos presentes y futuros, exteriores e interiores, en los hombres. Apreciarlo en sus grandes logros; entenderlo para ayudar a encauzarlo" 50 .

McGrath propone también en la ponencia otros dos signos: la valorización de lo temporal y de lo personal y el enfoque mundial (interdependencia, integración). El primero refiere, con distinta terminología y acentos, a la importante afirmación del Concilio sobre la autonomía de las realidades creadas y, el segundo, a la problemática que hoy se caracteriza como globalización ${ }^{51}$. En ese tratamiento, el autor no solo describe fenomenológicamente los acontecimientos, sino que ya insinúa las conexiones con temas teológicos.

En la conclusión afirma que "podrían haberse escogido otros entre los grandes signos de los tiempos en América Latina hoy", pero piensa que aquellos que podrían añadirse, como desarrollo, por ejemplo, de una u otra manera pueden incluirse en los escogidos. Unas frases de la conclusión caracterizan bien su perspectiva: "La rapidez y profundidad de los cambios que afectan a nuestros pueblos son el proceso histórico en que estas deficiencias han de entenderse (alude McGrath al egoísmo de los privilegiados, a la corrupción en la política, el militarismo); la creciente valorización de lo temporal caracteriza a la nueva situación, y el enfoque mundial es el marco nuevo y total que la acondiciona" ${ }^{52}$.

50 "Los signos de los tiempos", 80-81.

51 M. McGrath caracteriza al capítulo III de la I parte de Gaudium et spes como "el Corazón de la Constitución". Cf. "La génesis de Gaudium et spes", 502. Cf. M. McGrath, "A Living Theology in Latin America", 170: "Nuestras preocupaciones con el desarrollo y la liberación para nuestro pueblo han planteado, en un contexto latinoamericano, la preocupación teológica básica de Gaudium et spes: la relación entre el orden temporal y el eterno, de desarrollo y redención, de liberación e historia de la salvación" (cursiva mía). Esta constatación de McGrath debe ponerse en vinculación con lo que él considera "quizás el principal fenómeno político de Latinoamérica en esta generación", esto es, la "convicción” de que los "cristianos no pueden vivir divorciados de los asuntos temporales que tan profundamente afecta a su pueblo". McGrath afirma que esto difícilmente podía decirse de los católicos cincuenta o incluso treinta años antes. Cf. M. McGrath, "Ariel o Caliban?", en Foreign Affairs 52, 1 (1973) 75-95, 88. Este texto muestra que, si la ponencia de Medellín solo alude de modo general a los signos de los tiempos, a los procesos de transformación, McGrath posee amplios conocimientos históricos, políticos, económicos y culturales del continente, incluso en su relación con Estados Unidos.

52 M. McGrath, "Los signos de los tiempos", 96-97. 
La ponencia de McGrath es un texto digno de consideración, refleja de alguna forma el estado de la discusión del momento, también las perplejidades y las explicaciones insuficientes. Comparativamente, y quizás más allá de los textos en torno a Medellín, refleja una comprensión mayor que, no obstante, no ofrece un desarrollo y precisión conceptual como el de Chenu citado.

2.4. La ponencia sucesiva en la Conferencia, titulada "Interpretación cristiana de los signos de los tiempos hoy en América Latina”, correspondió a E. Pironio. Refiero aquí a ella solo como contrapunto o complemento a la de McGrath. El "hoy" de la salvación en el texto de Pironio no está puesto en relación a un signo de los tiempos en particular, sino más bien en referencia al momento histórico que vive el continente, que se juzga "providencial" ${ }^{3}$. A diferencia de la exposición de McGrath no hay en la ponencia del obispo argentino la búsqueda de clarificar la noción, tampoco emplea el concepto, salvo en el título, aunque la idea está recogida ya en su misma introducción (n.3).

Determinante para la comprensión del aporte de Pironio es el hecho de que esta ponencia no estaba pensada como reflexión sobre la primera parte del DT, el Ver, sino sobre la segunda, la reflexión teológica. De allí que, en su conjunto, el texto refleje un desarrollo más detallado de aquella parte del documento preparatorio. Como sucedió tanto con el DBP como con el DT, no falta una cierta circularidad hermenéutica. En este sentido no se exponen temas doctrinales simplemente yuxtapuestos a una realidad que no se explicita de alguna manera.

Si se tiene presente el conjunto de los textos de Medellín, un hecho llamativo del aporte de Pironio reside en que incluye 67 citas o referencias bíblicas (casi el doble que el documento final, con 37; 44 si se incluye la introducción y el mensaje) ${ }^{54}$; referencias, por lo demás, de ninguna manera ocasionales en la exposición del obispo argentino. Ellas verdaderamente impregnan el texto. Es de notar, sin embargo, que la ponencia no acude al texto neotestamentario donde, precisamente, aparece la

53 Aunque el texto comparte el diagnóstico de McGrath. Afirma E. Pironio: "Vivimos un momento particularmente grande en América Latina. Momento difícil y providencial. Su característica esencial es el cambio...”. Cf. "Interpretación cristiana de los signos de los tiempos hoy en América Latina”, en Celam, La Iglesia en la actual transformación, 101-122, 122.

54 Cf. J. O. Beozzo, “Medellín: inspiração e raízes”, 20. 
expresión: "semeia ton kairon" (Mt 16,3); referencia evangélica tampoco citada en el documento final. En este sentido, especialmente la ponencia de McGrath, en menor medida el silencio de Pironio, son índices claros de que "el lenguaje bíblico", "escatológico" del texto evangélico no ha sido integrado adecuadamente en la reflexión, de manera análoga a lo sucedido en el debate de Gaudium et spes ${ }^{55}$. Reflejan, probablemente, el estado de cuestión de la época. Tampoco el famoso texto de Chenu de 1965, el más importante en aquel momento, ofrece una conciencia del asunto.

\section{AlgunAS REFLEXIONES FINALES}

Formulo algunas reflexiones finales como fruto del itinerario recorrido.

La agenda de la Iglesia y de la Conferencia, a partir de la misma Introducción general del DBP, parece estar determinada completamente por la situación ad extra. Allí se advierte de la "tentación" de replegarse en la actuación "estrictamente eclesiástica y sacramental" con una "neutralidad que dejaría a otros la elaboración de la nueva cultura y de la nueva sociedad". No es de extrañar que esta percepción y este análisis conduzca a estructurar el tratamiento del temario de la Conferencia de una manera que no se repetirá en ninguna Conferencia general del episcopado posterior: en primer lugar, y de una forma comparativamente más extensa, la "promoción humana", en el sentido amplio y dependiente del magisterio conciliar que puede observarse en el documento final ${ }^{56}$. Lo advirtió con claridad M. McGrath: esta organización del material "altera el orden más frecuentemente usado en la Iglesia, antes y después de Medellín" ". Esa "alteración" dice mucho de Medellín, su peculiaridad y su diferencia, ya plenamente visible en las primeras páginas del DBP de enero de 1968.

Uno de los aspectos de los textos en torno a Medellín que admira, precisamente, es la pasión que reflejan por comprender la realidad en la

55 Puede matizarse el juicio sobre la exposición de Pironio si se advierten sus citas de Mc 1,5, Mt 12,28, 2 Co 6,2, etc.

56 Cf. C. SChickendantz, "Único ejemplo de una recepción continental del Vaticano II", en Teología 108 (2012) 25-53, 51-52.

57 "Algunas reflexiones sobre el impacto y la influencia permanente de Medellín", 165. 
que viven, el proceso histórico en el que están insertos en orden a intervenir en él en una línea de justicia y paz. Pero una limitación puede ser observada: para Pablo VI y el proceso de Medellín verificado en los textos que se han considerado el instrumento científico tiene un nombre casi exclusivo: la sociología. Los años siguientes complejizarían este punto de vista y favorecerían, progresivamente, una pluralidad de miradas multidisciplinares; tarea en plena realización que es posible comprobar en la bibliografía reciente. Por otra parte, los temores y reservas sobre el Ver y, en general, referidos al método jocista, "expresión de resistencia a una racionalidad inductiva e histórica" como ha afirmado A. Brighen$\mathrm{ti}^{58}, \tan$ visibles en las Conferencias siguientes, ocupan en Medellín un espacio mucho más modesto, casi inexistente.

La debilidad bíblica del método de Medellín, constatada más arriba, es probablemente deudora de la debilidad bíblica de la Constitución pastoral y de que el círculo hermenéutico Dei Verbum-Gaudium et spes es solo insinuado al final del Vaticano II, dejado como tarea al proceso de recepción posconciliar posterior. El Concilio que ofreció su gran adquisición metodológica, probablemente también contagió sus insuficiencias en la forma teológica de proceder. En particular los trabajos de C. Theobald en estos años muestran, precisamente, algunas de las limitaciones del paso adelante hecho en el Concilio, que es posible verificar también en los textos en torno a Medellín. Por otra parte, una contribución reciente de P. Suess ofrece un esbozo interesante y provocador del aporte que otorga una mirada bíblica, neotestamentaria, cristológica y escatológica de los signos de los tiempos, concretada además desde América Latina ${ }^{59}$. Tiempos mesiánicos desde un lugar de clase podría titularse su reflexión que, por contraste, desnuda las limitaciones de muchos de los textos analizados en este artículo.

El renombrado comentario de H.-J. Sander confirma completamente la lectura hecha por McGrath, más aún, aunque el teólogo alemán no se detiene particularmente en él, su comentario ayuda a comprender mejor la lucidez con la que el obispo panameño caracteriza el debate meto-

\footnotetext{
58 "Documento de Aparecida: o texto original, o texto oficial e o Papa Francisco", en Revista Pistis \& Práxis 8, 3 673-713, 690.

59 Cf. "Sinais dos tempos", en J. Decio Passos - W. Lopes Sanchez (coord.), Dicionário do Concílio Vaticano II (Paulinas, São Paulo 2015) 895-901.
} 
dológico conciliar del que había sido parte ${ }^{60}$. Si se tienen presente las discusiones conciliares y las posiciones de los diversos protagonistas de entonces (obispos y peritos) las breves pero acertadas observaciones de McGrath quedan mejor destacadas. Es verdad que falta un trabajo más sistemático en McGrath sobre el método y la categoría; simplemente no existe, pero en sus contribuciones en el contexto temporal de Medellín se explicitan algunos puntos clave en los que el obispo panameño parece ir más allá que muchos de los teólogos de su entorno. Es su punto y su mérito.

Las consideraciones de McGrath acerca del "doble nivel" de la categoría de signos de los tiempos, entre el "lenguaje bíblico" y la "otra forma, más actual", tiene mucho relieve a la luz de las discusiones de entonces. Indican, no obstante, un asunto metodológico necesitado de una clarificación conceptual, particularmente en lo que atañe a la articulación de los dos niveles ${ }^{61}$. La problemática se visibiliza, en especial, cuando se formula el efecto del círculo hermenéutico en la revelación misma. McGrath afirma en su ponencia: "es así, que los signos de los tiempos nos llevan a decir cosas nuevas del Evangelio", pero el límite de esa novedad se formula con claridad; "no en cuanto a él mismo, pero sí respecto de sus aplicaciones" ${ }^{62}$. No solo la densidad epistemológica de la praxis aparece como limitada en los textos analizados, sino también la más exacta naturaleza de la densidad teológica del presente. La pregunta por el estatuto teológico de esta "novedad" y de lo que ella significa para la metodología ocupa a la teología hasta hoy, permanece como una cuestión irresuelta ${ }^{63}$.

El itinerario recorrido en estas páginas converge con una constatación de V. Codina al evaluar la recepción del Vaticano II en

60 "Theologischer Kommentar zur Pastoralkonstitution über die Kirche in der Welt von heute Gaudium et spes", en P. Hünermann - B.-J. Hilberath (eds.), Herders Theologischer Kommentar zum Zweiten Vatikanischen Konzil. Band 4 (Herder, Freiburg i.Br. 2005) 581-886.

61 Los trabajos de G. Ruggieri y P. Hünermann, por citar algunos de los mejores, pueden mostrar el salto de calidad que se ha dado en estas décadas en la comprensión del doble nivel (ético-histórico y como fenómeno teológico). Cf. C. ScHicKENDANTZ, "Un cambio en la ratio fidei. Asociación (aparentemente ilícita) entre principios teológicos y datos empíricos", en Teología y Vida 57 (2016) 157-184, 174 ss.

62 "Los signos de los tiempos", 77 (cursivas mías).

63 Cf. C. Schickendantz, "Un cambio en la ratio fidei", 157-184. 
nuestro continente: Gaudium et spes ha sido el texto conciliar "que mayor impacto ha tenido en América Latina”, precisamente por el aporte de su método que invita a auscultar y discernir los signos de los tiempos en las aspiraciones y en los deseos profundos de la humanidad ${ }^{64}$. Los textos de Medellín y los aportes de McGrath analizados, sin decidir sobre el acierto o no de la formulación de Codina, revelan la profundidad de la vinculación entre la Constitución pastoral, la metodología implicada y el evento de Medellín.

Una convicción de McGrath, que también motiva este trabajo, estimula a profundizar en los múltiples asuntos pendientes: la conciencia que "del método depende mucho el resultado en cualquier proceso" 55 . Al cumplirse los 50 años de la Conferencia de Medellín parece oportuno explorar nuevamente el "secreto" (Beozzo) del que es, quizás, el acontecimiento más importante y más estimulante del siglo XX en la Iglesia de nuestro continente; "en cierto sentido, el acta de nacimiento de la Iglesia latinoamericana y caribeña" ${ }^{\circ 6}$.

64 Cf. "Las iglesias del continente 50 años después del Vaticano II", en Congreso Continental de Teología (ed.), 50 años del Vaticano II (Paulinas, Bogotá 2013) 8192,84 .

65 "Método teológico-pastoral: clave del posconcilio", en Teología y Vida 26 (1985) 285-293, 287.

66 J. O. Beozzo, “Medellin: Quarenta annos”, 1. 
